\title{
PROBLEMAS EN EDUCACIÓN SUPERIOR EN EL ESTADO DE VERACRUZ AL APLICAR PROGRAMACIÓN LINEAL
}

\section{PROBLEMS IN HIGHER EDUCATION IN THE STATE OF VERACRUZ WHEN APPLYING LINEAR PROGRAMMING}

\author{
E. Gómez Barrientos*, C. Martínez Galán, I. Hernández Palagot, \\ J.R. Ravell Delgado, G. Ocaña Medina \\ Tecnológico Nacional de México, Instituto Tecnológico Superior de San Andrés Tuxtla. División de \\ Ingeniería Industrial, San Andrés Tuxtla, Veracruz México. \\ *egb2407@hotmail.com
}

(recibido/received: 02-febrero-2020; aceptado/accepted: 11-mayo-2020)

\section{RESUMEN}

En investigación operativa, la técnica de programación lineal aplica diversos métodos para resolver modelos de programación matemática, se basa en álgebra matricial para llegar a la solución del problema, sin embargo, un problema que se presenta durante el proceso de optimización es la necesidad de trabajar con números racionales, lo que ocasiona que el desconocimiento para realizar las operaciones y el manejo inadecuado de las maquinas calculadoras provoque que la solución del problema en cuestión sea errónea. Este problema impacta en el aprendizaje de la asignatura de investigación de operaciones o afines, impartidas en los programas educativos de los Institutos Tecnológicos del Estado de Veracruz.

Palabras clave: Investigación operativa; Programación lineal; Programación matemática; Números racionales; Máquinas calculadoras.

\begin{abstract}
In operational research, the linear programming technique applies various methods to solve mathematical programming models, it relies on matrix algebra to arrive at the solution of the problem, however, a problem that arises during the optimization process is the need to work with rational numbers, which causes that the ignorance to carry out the operations and the inadequate handling of the calculating machines causes that the solution of the problem in question is wrong. This problem impacts on the learning of the operations or related research subject, taught in the educational programs of the Technological Institutes of the State of Veracruz.
\end{abstract}

Key words: Operational research; Linear programming; Mathematical programming; Rational numbers; Calculating machines. 


\section{E. Gómez Barrientos et al.}

\section{INTRODUCCIÓN}

En México, una de las materias en la que los estudiantes tienen mayor deficiencia son las matemáticas, principalmente en la solución de matrices y ecuaciones, siendo este un lenguaje simbólico que no asimilan la mayoría de los estudiantes de instituciones mexicanas de educación superior, de modo que un gran número de ellos eligen las carreras de ciencias sociales con la expectativa de tener menos contacto con las matemáticas, sin embargo en algunas de estas también se utilizan para la toma de decisiones.

Las matemáticas tienen por objetivo desarrollar habilidades en los estudiantes de cualquier nivel educativo, requiriendo del uso de estrategias que permitan desarrollar las capacidades para entender, asociar, analizar e interpretar los conocimientos adquiridos para afrontar su entorno, dentro de las cuales se encuentran los números fraccionarios que permiten resolver diversas situaciones de la vida cotidiana pero que mayor parte de la población estudiantil presenta problemas al hacer uso de estas a pesar de que el estudio de fracciones es enseñado desde las primeras etapas educativas.

El presente artículo surge por la necesidad de identificar la dificultad del uso de operaciones fraccionarias en la resolución de problemas con el Método Simplex a través de análisis de los factores que afectan el aprendizaje y la práctica de operaciones de esta índole. De acuerdo con la investigación realizada en este ámbito, no existe Institución de Educación Superior (IES) que no se enfrente a esta problemática de reprobación y bajo rendimiento académico.

Actualmente existe mucha inquietud por parte de los docentes acerca de la problemática existente en la enseñanza de las fracciones en los distintos niveles educativos, puesto que el fracaso escolar está presente en cualquier institución educativa, obteniendo como consecuencia el incremento del índice de reprobación. Morales (1999) expone que la reprobación como parte del fracaso escolar es preocupante en todos los niveles educativos, pero que a nivel de licenciatura adquiere una importancia mayúscula, ya que de este nivel egresan los profesionales que deben incorporarse al aparato productivo nacional, para así contribuir al desarrollo del país.

\section{METODOLOGÍA}

Este estudio se realizó bajo los principios del método combinado basado en la recopilación de hallazgos de estudios de caso para responder a preguntas de una evaluación que pueden ser descriptivas, normativas o de causa y efecto, para efectos de esta investigación se utilizó el método inductivo-deductivo, en el cual según Bernal Torres (2010) se basa en la lógica y estudio de hechos particulares. Se incluye dos tipos de investigaciones en el desarrollo de la misma, de acuerdo con Serradó Bayés, Azcárate Góded, \& Cardeñoso Domingo (2009) a lo largo de todo el proceso de resolución de problemas, los alumnos desarrollan una serie de destrezas que requieren previamente la adquisición de capacidades, numéricas y digitales, mismas que se indagarán iniciando con una investigación documental sobre educación de Nivel Superior, dominio de fracciones, rendimiento académico, entre otros; y una investigación de campo para identificar la dificultad en el uso de operaciones con fracciones en la resolución de problemas con el Método Simplex, misma que se desarrolló mediante una encuesta a alumnos del Estado de Veracruz de diversos Tecnológicos pero no todos integran en los planes de estudio de sus carreras la materia de Investigación de Operaciones o Métodos Cuantitativos, las cuales contemplan en su programa el Método Simplex, por tanto, esta investigación se centró principalmente en 29 Tecnológicos del Estado de Veracruz.

Bowley (2005) afirma que no siempre es necesario obtener completa información de todos los miembros de un grupo para dar cuenta adecuada del mismo, por tanto, con la ecuación 1 se obtuvo una muestra finita 


\section{E. Gómez Barrientos et al.}

$n$ para determinar a qué Tecnológicos se aplicaría el instrumento para obtener datos reales acerca del problema de investigación, aplicando la respectiva fórmula para dicho calculo:

$$
n=\frac{Z_{1-\alpha * \mathrm{p} * \mathrm{q} * \mathrm{~N}}^{2}}{d^{2} *(N-1)+Z_{1-\alpha}^{2} * p * q}
$$

Así, se obtuvo una muestra de 24 Tecnológicos en el Estado, posteriormente se determinó a cuantas personas se aplicaría el instrumento. Para calcular este dato, se ocupó la fórmula de estimación de muestra infinita, dada la resolución de dicha fórmula se estableció que el total de alumnos a encuestar en los diversos Tecnológicos seleccionados sería de 384 alumnos.

Una vez determinado a cuantos Tecnológicos y a cuantas personas en general se aplicarían las encuestas, se realizó un muestreo por cuotas, el cual según Mendieta Izquierdo (2015) es definido por características, parámetros biológicos o socio demográficos; teniendo como base, que solo se encuestarían a alumnos que hubiesen cursado alguna de las materias mencionadas con anterioridad; teniendo 384 encuestas y 24 Tecnológicos, se seleccionó de forma equitativa los alumnos a encuestar de cada tecnológico teniendo como resultado un total de 16 alumnos por tecnológico como muestra representativa de la materia de Investigación de Operaciones y Métodos Cuantitativos.

\subsection{PARTICIPANTES}

Los participantes del estudio se han seleccionado mediante un muestreo aleatorio. La muestra está constituida por 384 estudiantes universitarios tomados de los diferentes tecnológicos del estado de Veracruz, en cada uno se aplicaron 16 cuestionarios, los jóvenes se eligieron de manera aleatoria y de diferentes carreras. Ver figura 1.

\section{Institución}

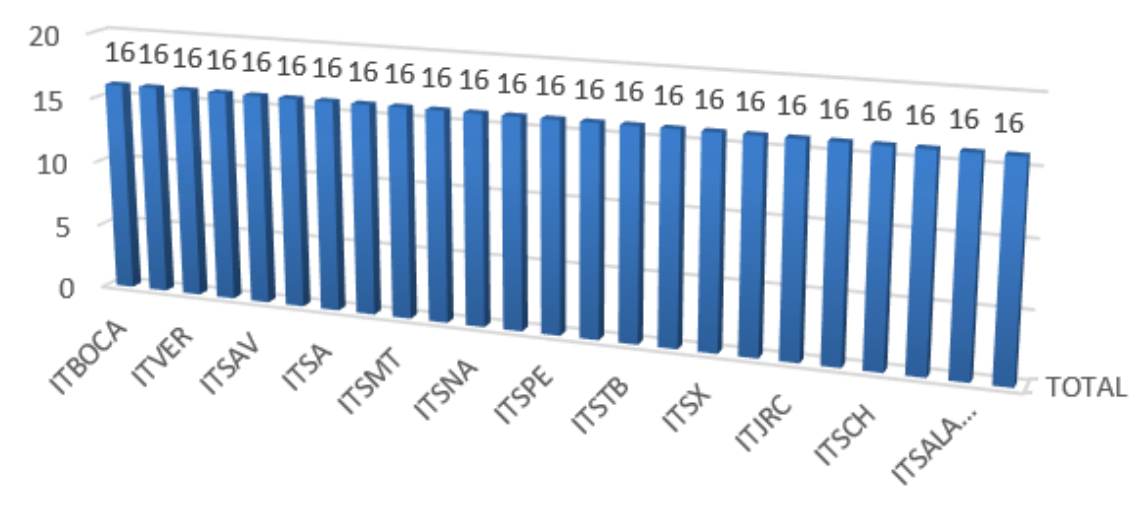

Figura 1. Gráfico de Institutos Tecnológicos que se tomaron en cuenta para el estudio.

De estas instituciones los encuestados fueron jóvenes que cursan 6 diferentes carreras, pero tienen en común que su curricula contempla la materia de Investigación de operaciones o Métodos cuantitativos, siendo parte de la muestra aquellos jóvenes que estudian de cuarto semestre en adelante, porque ellos ya cursaron la materia mencionada o la están cursando. En la figura 2 se presentan los programas o carreras considerados por contar en su mapa curricular con la asignatura. 


\section{E. Gómez Barrientos et al.}

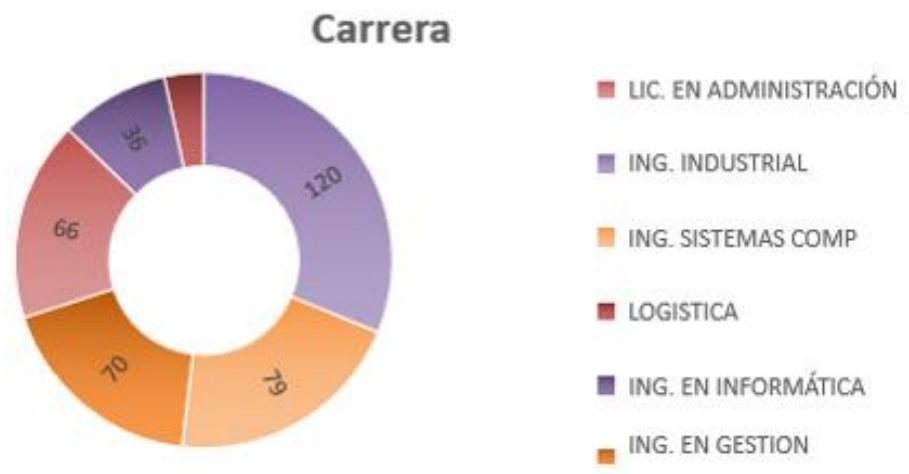

Figura 2. Programas de estudio considerados en la investigación y jóvenes que se tomaron por programa

\section{RESULTADOS}

De acuerdo con la información proporcionada por estudiantes de Tecnológicos del Estado de Veracruz, se obtuvieron datos relevantes acerca de las inquietudes manifestadas a lo largo del desarrollo de la investigación, lo cual contempla desde conceptos manejados en Investigación de Operaciones-Métodos Cuantitativos hasta la complejidad de resolución del Método Simplex, mismo que se relaciona con el desarrollo de operaciones con números fraccionarios; cabe mencionar que el enfoque de la encuesta realizada es fundamentar la dificultad de operaciones realizadas en el Método Simplex.

\section{Del apartado I del cuestionario en el manejo de conceptos y operaciones fundamentales se obtuvieron}

\section{lo siguiente: Dificultad}

Cuando se cuestionó a los jóvenes si entendían los conceptos manejados en la Investigación de operaciones, quedo de manifiesto que $26 \%$ de los jóvenes si entendía, el $68 \%$ respondió que solo entendía un poco, y el resto respondió que no entendía nada. Esta información se presenta en la gráfica de la figura 3.

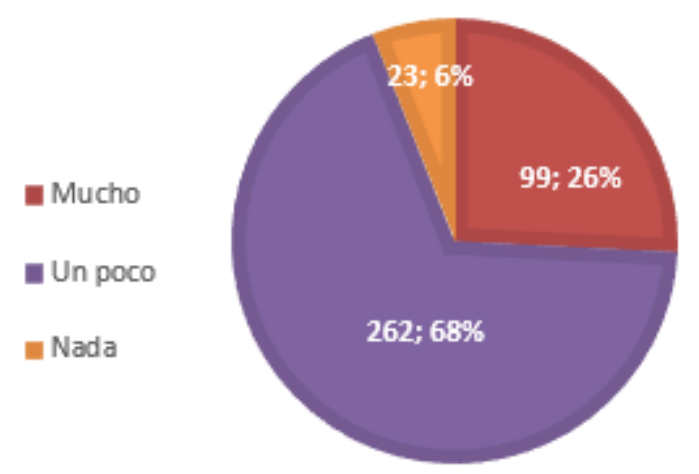

Figura 3. Representación gráfica de repuestas a la pregunta 1

Al preguntar ¿Dominas operaciones con fracciones? El $24.74 \%$ contesto que si tenían dominio de estas operaciones, el $63.54 \%$ dijo que solo un poco y el $11.72 \%$ dijo que nada. Ver figura 4. 


\section{E. Gómez Barrientos et al.}

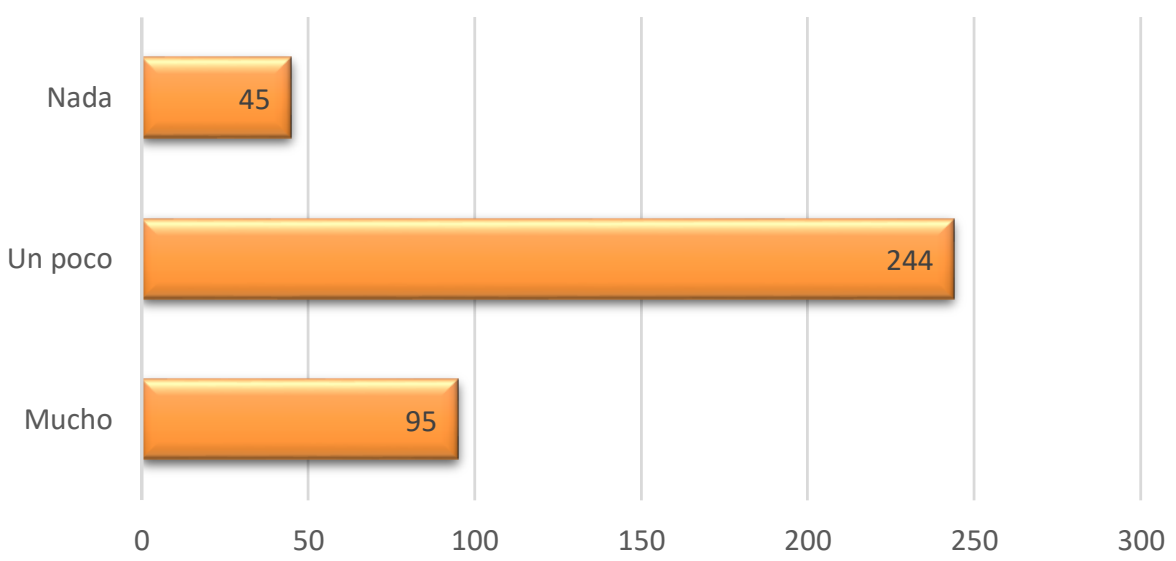

Figura 4. Gráfico que muestra número de jóvenes que manifestaron tener dominio de operaciones con fracciones

Cuando se les cuestiono sobre el dominio de operaciones matriciales, el $22.3 \%$ dijo dominar las operaciones matriciales, $63.5 \%$ respondido que solo un poco y $11.71 \%$ contestó que no dominan estas operaciones. En la figura 5 se aprecia la cantidad de alumnos y el dominio con operaciones matriciales.

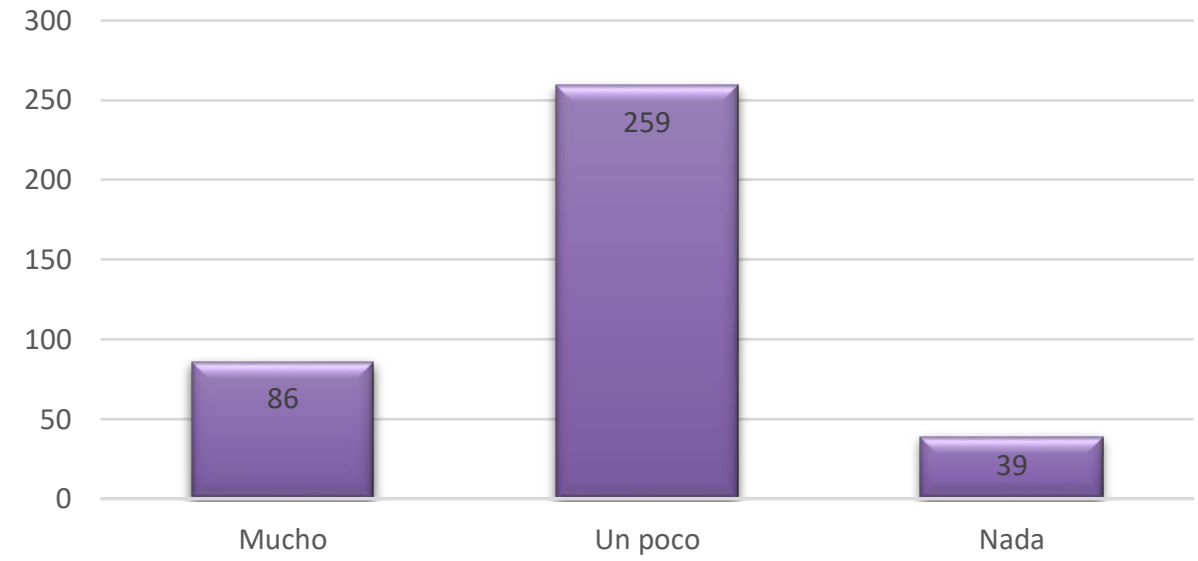

Figura 5. Gráfico que muestra número de jóvenes que manifestaron tener dominio de operaciones matriciales

Del apartado II del cuestionario: Dificultades en operaciones por renglón, se cuestionó sobre las asignaturas de Investigación de operaciones / Métodos cuantitativo según la que hayan cursado, ¿Cuál método consideras que fue el más complicado? El 73.95\% de los jóvenes contestaron que el método más complicado fue el método simplex, $19.79 \%$ comento que el método gráfico, el 3.65\% respondió que el método de trasporte y $11.97 \%$ considera que todos son complicados. Como se aprecia en la figura 6 el método simplex resulta ser el método más complicado para los alumnos. 


\section{E. Gómez Barrientos et al.}

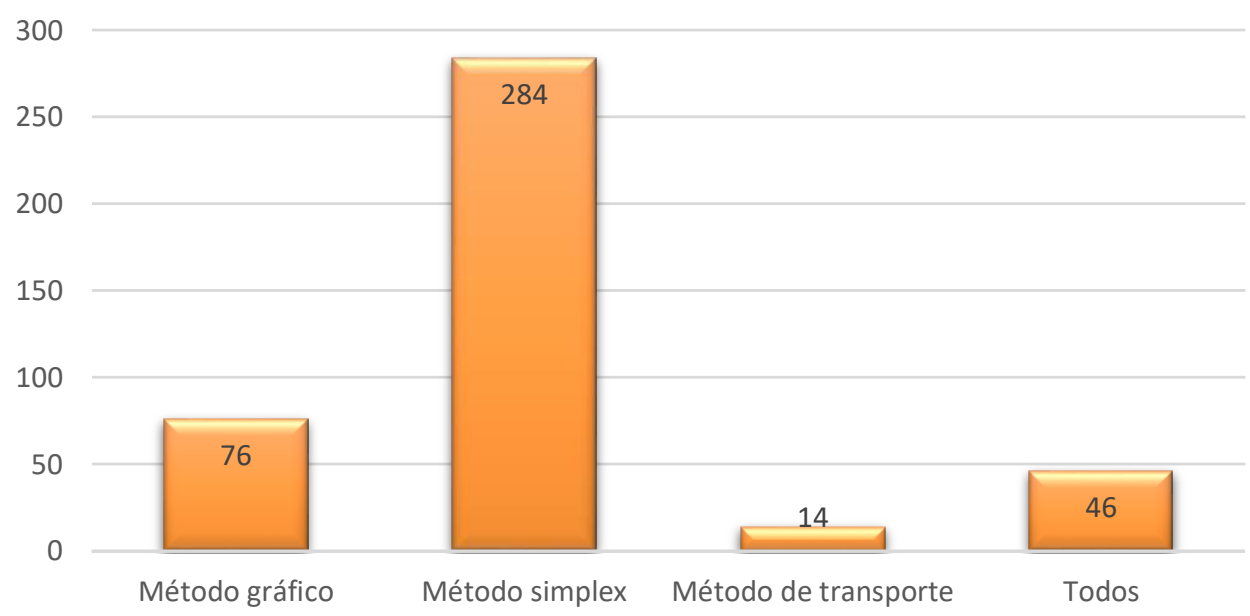

Figura 6. Gráfico que muestra que para los estudiantes el método simplex es el más complicado de la Investigación de operaciones

Cuando se les cuestionó a los jóvenes sobre ¿Qué problemas presentas a la hora de realizar las operaciones en el método simplex?, el 52\% respondió que la mayor dificultad está en las operaciones con quebrados, el $17.44 \%$ menciona que, en las técnicas de enseñanzas, el 5.98\% coinciden que la dificultad está en el uso de la calculadora y el $24.22 \%$ considera que la dificultad está en trabajar con números negativos. La figura 7 presenta la información que arroja que los números racionales son el mayor problema para los alumnos.

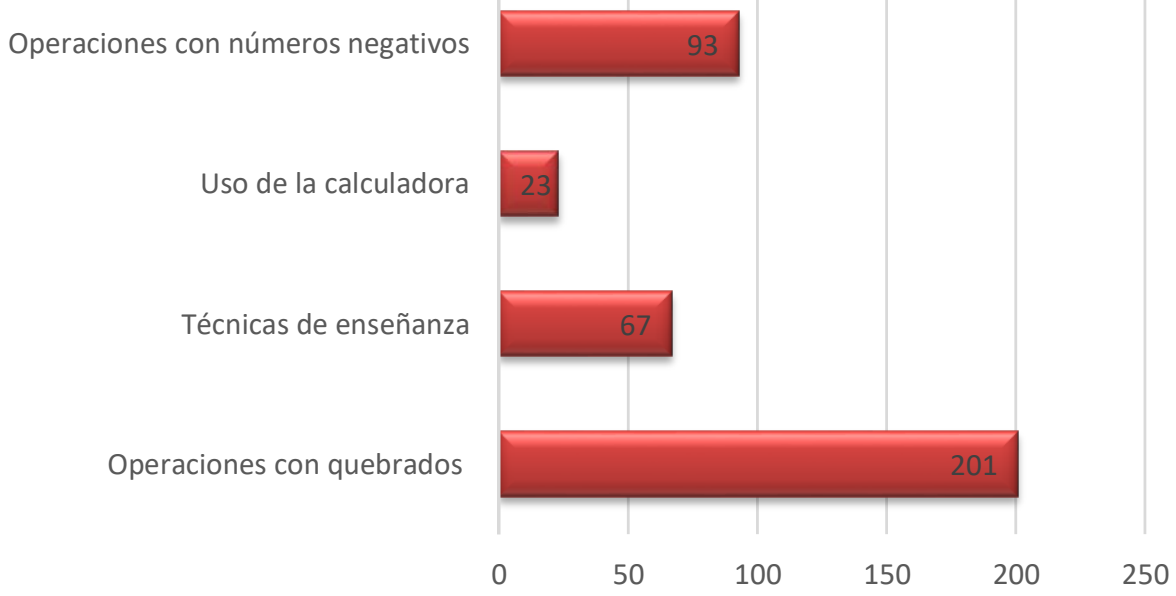

Figura 7. Representación Gráfica de los problemas que enfrentan los jóvenes al realizar el método simplex

De acuerdo a la pregunta 8 que se realizó en el cuestionario aplicado a los alumnos de los Tecnológicos del Estado, en la cual se preguntó ¿Qué tan complicado es realizar operaciones por renglón en el Método Simplex?, se obtuvo que $40.36 \%$ contesto que es medio complicado, $30.46 \%$ dijo que es complicado, $10.67 \%$ respondido que es muy complicado, $15.36 \%$ dijo que son poco complicadas y $3.1 \%$ menciono que no es complicado. Ver figura 8. 


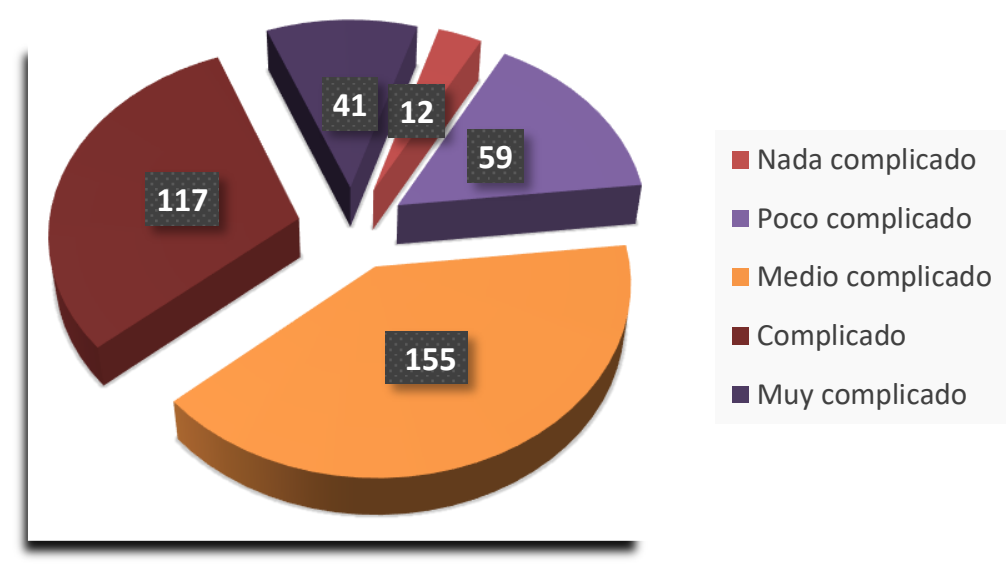

Figura 8. Gráfico que muestra la percepción de los alumnos sobre el grado de complejidad de las operaciones de renglón.

Una vez se concentraron los resultados de las encuestas aplicadas, se determinaron algunas aseveraciones importantes referentes a las instituciones educativas que se tomaron en cuenta para el análisis, para ello se definieron zonas en las cuales están ubicados los tecnológicos del estado de Veracruz.

Tabla 1. Concentrado por región de las respuestas a las preguntas 1,2, 3 .

\begin{tabular}{|c|c|c|c|c|}
\cline { 2 - 5 } \multicolumn{1}{c|}{} & \multicolumn{3}{c|}{ Dificultad en el manejo de conceptos y operaciones fundamentales } \\
\cline { 2 - 5 } \multicolumn{1}{c|}{} & \multicolumn{3}{c|}{ Región } & \multirow{2}{*}{ Total } \\
\hline Pregunta & Norte & Centro & Sur & \\
\hline 1 & $42.71 \%$ & $27.60 \%$ & $23.70 \%$ & $94.01 \%$ \\
\hline 2 & $39.58 \%$ & $25.78 \%$ & $22.92 \%$ & $88.28 \%$ \\
\hline 4 & $40.10 \%$ & $26.82 \%$ & $22.92 \%$ & $89.84 \%$ \\
\hline
\end{tabular}

Respecto a la tabla 1, la pregunta 1 cuestiona lo siguiente: ¿Entiendes los conceptos manejados en la Investigación de Operaciones?, en la región Norte se refleja que un $42.71 \%$ no dominan en absoluto los conceptos manejados. La pregunta 2 corresponde a: ¿Dominas operaciones con fracciones?, se manifiesta que en el Norte el 39.58\% no lo domina y finalmente en la pregunta 4: ¿Dominas operaciones matriciales? se expresa que en el Norte un $40.10 \%$ no puede dominar las operaciones matriciales siendo el porcentaje más alto a diferencia de las otras regiones; además cabe mencionar que la región Norte cuenta con 11 Tecnológicos, mientras que el Centro cuenta con 7 y el Sur con 6.

Cuando se preguntó a los jóvenes sobre la materia de investigación de operaciones, ¿Cuál método consideras que fue el más complicado? los jóvenes coincidieron que el método simplex.

¿Qué problemas presentas a la hora de realizar las operaciones en el método simplex? En las tres regiones los jóvenes coincidieron en que los problemas a los que se enfrentaba eran las operaciones con quebrados 


\section{E. Gómez Barrientos et al.}

Tabla 2. Categorización por región de la problemática para realizar operaciones

\begin{tabular}{|c|c|c|c|c|}
\cline { 2 - 5 } \multicolumn{1}{c|}{} & \multicolumn{4}{c|}{ Dificultad en operaciones por renglón en el Método Simplex } \\
\cline { 2 - 5 } \multicolumn{1}{c|}{} & \multicolumn{3}{c|}{ Total } \\
\hline Pregunta & Norte & Cenión & \\
\hline 7 & $22.5 \%$ & $35 \%$ & $42.5 \%$ & $100 \%$ \\
\hline
\end{tabular}

De acuerdo a la pregunta 8 que se realizó en el cuestionario aplicado a los alumnos de los Tecnológicos del Estado, en la cual se preguntó ¿Qué tan complicado es realizar operaciones por renglón en el Método Simplex?, se obtuvo que la región donde más dificultad existe para calcular dichas operaciones es en la región Sur del Estado.

\section{CONCLUSIONES}

En todo el Estado de Veracruz se puede visualizar que de los 384 encuestados el $94.01 \%$ se les dificultad el manejo de los conceptos aplicados a Programación Lineal ocasionando un problema en su enseñanza, puesto que además de no comprender los conceptos que se manejan se tiene que el $88.28 \%$ no sabe realizar operaciones con fracciones, lo que provoca una dificultad en el dominio de operaciones matriciales con un $89.84 \%$ de los estudiantes encuestados, además se sabe que las fracciones son fundamentales para su desarrollo y esto provoca que los resultados obtenidos en la resolución del Método Simplex sean erróneos, generando frustración a los estudiantes que no saben realizar operaciones con fracciones.

Como puede observarse el $81.51 \%$ de los encuestados expresan que existe dificultad en la realización de operaciones de reglón, por lo que se concluye que el uso de operaciones con fracciones es un factor perjudicial que afecta el proceso de aprendizaje en estudiantes de educación superior, pues de acuerdo con los resultados, existe un porcentaje elevado de mal manejo y comprensión de los modelos de Programación Lineal además de que les resulta difícil el realizar operaciones con fracciones las cuales son empleadas para la solución de problemas dentro del Método Simplex.

Es evidente entonces que el principal problema detectado al momento que el alumno realiza sus operaciones para dar respuesta a un modelo de programación lineal se encuentra el mal manejo de operaciones con fracciones, esto debido a que los alumnos no saben realizar operaciones fraccionarias teniendo como efecto la deserción de los mismos en la materia de Investigación de Operaciones, específicamente en la aplicación del Método Simplex, de manera que se propone un método alternativo, como lo es el llamado Montante para la resolución de problemas a partir de operaciones con número enteros. 


\section{E. Gómez Barrientos et al.}

\section{REFERENCIAS}

Bernal Torres, C. A. (2010). Metodología de la investigación. Colombia: Pearson.

Bowley, A. L. (2005). Muestreo (An elementary manual of statistics). EMPIRIA. Revista de Metodología de las Ciencias Sociales, 217-224.

Freudenthal, H. (1973). Mathematics as an Educational Task. Paises Bajos: D. Reidel Publishing Company.

Godino, J. D. (2014). Indicadores de la idoneidad didáctica de procesos de enseñanza y aprendizaje de las matemáticas. Cuadernos de Investigación y Formación en Educación Matemática. Portal de revistas académicas. https://revistas.ucr.ac.cr/index.php/cifem/article/view/14720

González del Olmo, D. (2015). Errores comunes en el aprendizaje de las fracciones: Un estudio con alumnos de 12/13 años en Cantabria. UCrea Repositorio Abierto de la Universidad de Cantabria. http://hdl.handle.net/10902/6903

Mendieta Izquierdo, G. (2015). Informantes y muestreo en investigación cualitativa. Investigaciones Andina, $17(30), 1148-1150$

Morales G.J. 1999. "La reprobación como factor del fracaso escolar" Memorias. Universidad Juárez Autónoma de Tabasco. Secretaría de Servicios Académicos. Ed. Edgo. Servicios Educativos.

Ramírez Leal, A. L., Buitrago Suescún, O. Y., Britto Agudelo, R. A. (2012) Un nuevo algoritmo para la solución de problemas de programación lineal. Ingeniería e Investigación, 68-73.

Saucedo Fernández, M., Herrera-Sánchez, S. d., Díaz Perera, J, J., Bautista Maldonado, S., \& Salinas Padilla, H. A. (2014). Indicadores de reprobación: Facultad de Ciencias Educativas (UNACAR). RIDE Revista Iberoamericana para la Investigación y el Desarrollo Educativo, 5(9). 96-106.

Serradó Bayés, A., Azcárate Góded, P., \& Cardeñoso Domingo, J. M. (2009). "NUMBERS: ZONA CERO" (I): MÉTODO CIENTÍFICO DE INVESTIGACIÓN ESTADÍSTICA. Revista Eureka sobre Enseñanza y Divulgación de las Ciencias, 6(1), 47-62.

\section{SEMBLANZA DE LOS AUTORES}

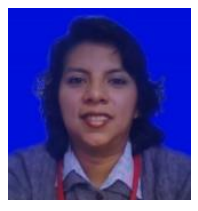

Elvira Gómez Barrientos: Obtuvo el grado de Ingeniero Industrial en el Instituto Tecnológico Superior de San Andrés Tuxtla, donde actualmente es Jefe de la División de Ingeniería Industrial. Desarrolló sus estudios de Maestría en Ingeniería Industrial en la Universidad Cristóbal Colon. Se ha desarrollado como docente del programa de Ingeniería Industrial con asignaturas como Administración de Operaciones, Investigación de Operaciones y Relaciones Industriales entre otras,

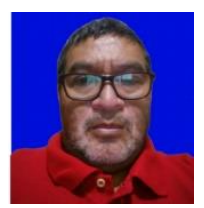

Carlos Martínez Galán: Obtuvo el grado de Ingeniero Mecánico en el Instituto Tecnológico de Orizaba, actualmente es docente del programa de Ingeniería Industrial en el Instituto Tecnológico Superior de San Andrés Tuxtla. Desarrolló sus estudios de Maestría en Ciencias en Ingeniería Industrial en el Instituto Tecnológico de Orizaba. Se ha desarrollado como docente del programa de Ingeniería Industrial con asignaturas como Investigación de Operaciones, Simulación, Automatización de procesos industriales entre otras.

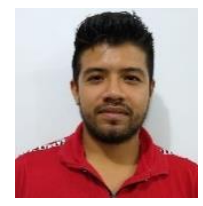

Israel Hernández Palagot: Estudiante de octavo semestre de la Carrera de Ingeniería Industrial en el Instituto Tecnológico Superior de San Andrés Tuxtla, integrante de la primera generación del programa PISI (Programa Institucional de Semilleros de Investigación) del Instituto Tecnológico Superior de San Andrés Tuxtla 


\section{E. Gómez Barrientos et al.}

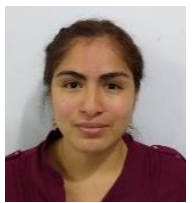

Jennifer Rosario Ravell Delgado: Estudiante de octavo semestre de la Carrera de Ingeniería Industrial en el Instituto Tecnológico Superior de San Andrés Tuxtla, integrante de la primera generación del programa PISI (Programa Institucional de Semilleros de Investigación) del Instituto Tecnológico Superior de San Andrés Tuxtla.

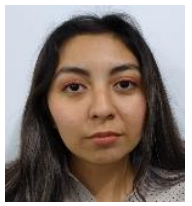

Gabriela Ocaña Medina: Estudiante de octavo semestre de la Carrera de Ingeniería Industrial en el Instituto Tecnológico Superior de San Andrés Tuxtla, integrante de la primera generación del programa PISI (Programa Institucional de Semilleros de Investigación) del Instituto Tecnológico Superior de San Andrés Tuxtla. 